\title{
Correlation of Nuclear Morphometry with Clinicopathologic Parameters in Malignant Breast Aspirates
}

\author{
Shivani Kalhan ${ }^{1}$ Shilpa Garg ${ }^{1}$ Rahul N. Satarkar ${ }^{1} \quad$ Puja Sharma ${ }^{1}$ Sonia Hasija ${ }^{1}$ Sonia Sharma ${ }^{2}$ \\ ${ }^{1}$ Department of Pathology, Shaheed Hasan Khan Mewati \\ Government Medical College, Nalhar, Haryana, India \\ ${ }^{2}$ Department of Pathology, Army Hospital (Research \& Referral), \\ New Delhi, India

\begin{abstract}
Address for correspondence Shilpa Garg, MBBS, MD Pathology, Department of Pathology, Shaheed Hasan Khan Mewati Government Medical College, Nalhar, Haryana, India
\end{abstract} \\ (e-mail: shilpagoyal15@gmail.com).
}

South Asian J Cancer 2021;10:64-68.

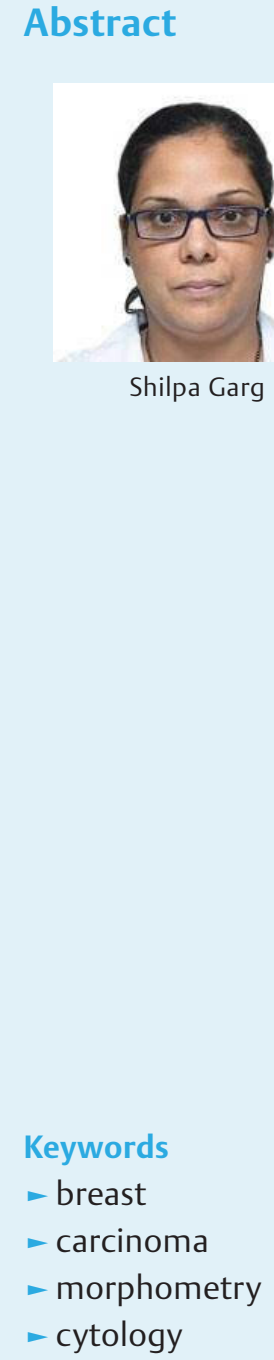

Objectives The primary objective of this study was to correlate nuclear morphometric parameters with clinicopathologic features such as cytologic grade, tumor size, lymph node status, mitotic index, and histopathologic grade. Secondary objective was to quantify nuclear changes on malignant breast aspirates using morphometry.

Material and Methods Forty-five cases of carcinoma breast diagnosed on cytology were included in this study. These were graded into cytologic grades 1, 2, and 3 as per Robinson's cytologic grading system.

Nuclear morphometry was done in all cases on smears stained with Papanicolaou stain. Clinicopathologic parameters including cytological grade, tumor size, lymph node status, mitotic count, and histological grade were correlated with nuclear morphometric parameters, namely, area, perimeter, shape, long axis, short axis, intensity, long-run emphasis, total run length, and $\mathrm{T} 1$ homogeneity.

Results There were 9 cases in cytologic grade 1, 26 in grade 2, and 10 cases in cytologic grade 3. Histopathology showed 42 cases of infiltrating duct carcinoma, not otherwise specified (IDC, NOS) and 3 cases (6.7\%) of ductal carcinoma in situ (DCIS). IDC (NOS) included 6,27 , and 9 cases in grades 1,2 , and 3 , respectively. Majority of our cases had a tumor size less than $5 \mathrm{~cm}(n=38,84.4 \%)$ and had positive nodes $(n=30,66.7 \%)$. Correlation of cytologic and histopathologic grades (including DCIS) with all morphometric features except long-run emphasis was statistically significant. Correlation of morphometry with tumor size yielded significant results for nuclear area, perimeter, long and short axes, and intensity with $p<0.05$. Study of lymph node status (positive/negative) versus morphometry showed a highly significant statistical association with all the geometric as well as textural parameters. Mitotic count was significantly associated with all the geometric parameters and one textural parameter (total run length).

Statistics Continuous variables were presented as mean \pm standard deviation and compared using the two-tailed, independent sample $t$-test and one-way analysis of variance test. Tests were performed at significance level of 0.05 .

Conclusion Morphometry is an objective technique which holds immense promise in prognostication in breast carcinoma.
DOI https://doi.org/10.1055/s-0041-1731583 ISSN 2278-330X

How to cite this article: Kalhan S, Garg S, Satarkar R. N, Sharma P, Hasija S, Sharma S. Correlation of Nuclear Morphometry with Clinicopathologic Parameters in Malignant Breast Aspirates South Asian J Cancer 2021;10(2):64-68.
(C)2021. MedIntel Services Pvt Ltd.

This is an open access article published by Thieme under the terms of the Creative Commons Attribution-NonDerivative-NonCommercial-License, permitting copying and reproduction so long as the original work is given appropriate credit. Contents may not be used for commercial purposes, or adapted, remixed, transformed or built upon. (https://creativecommons.org/licenses/by-nc-nd/4.0/).

Thieme Medical and Scientific Publishers Private Ltd A-12, Second Floor, Sector -2, NOIDA -201301, India 


\section{Introduction}

Alterations in nuclear structure are the morphologic hallmark of cancer diagnosis. Nuclear size, shape, chromatin pattern, and nucleolar size and number have all been reported to change in breast cancer. Attempts to quantify nuclear alterations to establish grading systems, predict prognosis, and/or set guidelines for therapy have met with varied success. ${ }^{1}$

Prognosis of breast cancer depends on multiple clinicopathological parameters which include tumor size, lymph node status, estrogen receptor status, tumor histologic grading, cell proliferation index, etc. These parameters are studied on surgically excised specimens. Treatment decisions are taken by the clinicians depending on these factors. Fine needle aspiration cytology (FNAC) is routinely used as a preoperative diagnostic tool in suspected cases of breast cancer. Information obtained by fine needle aspiration can be extremely useful in patient management.

This study was planned with the aim to quantify nuclear changes on malignant breast aspirates using morphometry and to correlate the morphometric parameters with clinicopathologic features such as cytologic grade, tumor size, lymph node status, mitotic index, and histopathologic grade.

\section{Material and Methods}

The present study was prospective in nature and commenced after due clearance from institutional ethics committee. Forty-five cases of carcinoma breast diagnosed on cytology were included. These were subsequently categorized on histopathology as 42 cases of infiltrating duct carcinoma, not otherwise specified (IDC [NOS]) and 3 cases of ductal carcinoma in situ (DCIS). A concise clinical history, examination, and details of relevant investigations like mammography were also obtained.

All cases of carcinoma breast, diagnosed on cytology were included in the study. On the other hand, patients who were lost to follow-up after cytologic diagnosis, that is, those for whom histopathologic diagnosis was not available, and those who were given preoperative neoadjuvant radiotherapy or chemotherapy were excluded from the present study.

FNAC was performed using standard procedure. Both air-dried and alcohol-fixed smears were stained by Leishman-Giemsa and Papanicolaou (PAP) stains, respectively. Cytologic grading was performed as per Robinson's cytologic grading system considering six parameters, namely, cell dissociation, cell size, cell uniformity, nucleoli, nuclear margin, and chromatin.

Scores of 1 to 3 were assigned for each of the six parameters-cell dissociation, cell size, cell uniformity, nucleoli, nuclear margin, and chromatin-and they were totaled to classify the lesions into: grade 1 , score 6 to 11 ; grade 2 , score 12 to 14 ; and grade 3 , score 15 to $18 .^{2}$

Nuclear morphometry was done in all cases on smears stained with PAP stain using Defense Bioengineering and Electromedical Laboratory cytoscan indigenously developed by the Defense Research and Development Organization, New Delhi, India. One hundred nonoverlapping cells per case were evaluated. Both geometrical and textural parameters were evaluated. Geometrical parameters included nuclear area, perimeter, nuclear shape, long axis, short axis, and intensity. Textural parameters were long-run emphasis (measuring coarseness of nuclear chromatin), total run length (measuring proportion of coarse to fine chromatin), and T1 homogeneity (measuring homogeneity of chromatin distribution).

Cytological grade, tumor size, lymph node status, mitotic count, and histological grade were correlated with nuclear area, perimeter, nuclear shape, long axis, short axis, intensity, long-run emphasis, total run length, and T1 homogeneity and it was studied that if the association was statistically significant.

Statistical analysis was performed using Statistical Package for the Social Sciences (SPSS) software for Windows, version 17.0 (SPSS, Chicago, Illinois, United States). Continuous variables were presented as mean \pm standard deviation (SD) and compared using the two-tailed, independent sample $t$-test and one-way analysis of variance test. Tests were performed at significance level 0.05 , that is, $p$-value less than 0.05 was taken to indicate a significant difference.

\section{Results}

The study included 45 cases of carcinoma breast diagnosed and graded on cytology. There were 44 females and 1 male patient. The mean age at diagnosis across the groups was 49.8 \pm 13.2 years and that in the DCIS group was $35.3 \pm 1.5$ years; IDC (NOS) with negative nodes was $50.3 \pm 13.5$ years and IDC (NOS) with positive nodes was $51.2 \pm 12.9$ years.

Histopathology was available in all 45 cases.

There were 9 cases in cytologic grade 1, 26 in grade 2, and 10 cases in cytologic grade 3. Correlation of nuclear morphometry with cytologic grades was performed. It was found that nuclear area, perimeter, shape, long axis, short axis, intensity, total run length, and T1 homogeneity were highly significant on statistical analysis ( - Table 1 ).

Majority of our cases had a tumor size less than $5 \mathrm{~cm}$ $(n=38,84.4 \%)$. There were seven cases with tumor size $\geq$ $5 \mathrm{~cm}$. Comparison of morphometric features with tumor size yielded significant results for nuclear area, perimeter, long and short axes, and intensity with $p<0.05$ for these parameters ( - Table 2).

There were 30 cases (66.7\%) with positive lymph nodes. Evaluation of lymph node status (positive/negative) versus morphometry showed a highly significant association between all the geometric as well as textural parameters, that is, nuclear area, perimeter, shape, long and short axes, intensity, long-run emphasis, total run length, and T1 homogeneity ( - Table 3 ). The three cases of DCIS were grouped in the node negative category since none of them had positive lymph nodes. Mitotic count was, on the other hand, significantly associated with geometric parameters such as nuclear area, perimeter, shape, long axis and short axis, and intensity. Among the textural parameters, only total run length, signifying proportion of coarse to fine chromatin, was found to be significantly associated with mitotic count on statistical analysis. Majority of our cases were in the $<6$ mitoses category ( $n=33,73.3 \%$; - Table 4$)$. 
Table 1 Correlation of cytologic grades with nuclear morphometry grades

\begin{tabular}{|l|l|l|l|l|}
\hline $\begin{array}{l}\text { Morphometric } \\
\text { parameter }\end{array}$ & $\begin{array}{l}\text { Cytology grade } \mathbf{1} \\
\boldsymbol{n}=\mathbf{9}\end{array}$ & $\begin{array}{l}\text { Cytology grade } \mathbf{n} \\
\boldsymbol{n}=\mathbf{2 6}\end{array}$ & $\begin{array}{l}\text { Cytology grade } \mathbf{3} \\
\boldsymbol{n}=10\end{array}$ & $\begin{array}{l}\boldsymbol{p} \text {-Value } \\
\text { (one-way ANOVA test) }\end{array}$ \\
\hline Nuclear area & $78.21 \pm 12.40$ & $93.12 \pm 13.85$ & $115.89 \pm 19.03$ & $<0.001^{\mathrm{a}}$ \\
\hline Perimeter & $31.08 \pm 4.24$ & $36.81 \pm 3.77$ & $39.33 \pm 5.32$ & $<0.001^{\mathrm{a}}$ \\
\hline Shape & $1.05 \pm 0.01$ & $1.09 \pm 0.04$ & $1.10 \pm 0.03$ & $0.005^{\mathrm{a}}$ \\
\hline Long axis & $11.94 \pm 0.97$ & $13.70 \pm 1.26$ & $14.82 \pm 1.19$ & $<0.001^{\mathrm{a}}$ \\
\hline Short axis & $8.24 \pm 0.81$ & $9.33 \pm 1.12$ & $10.20 \pm 0.95$ & $0.001^{\mathrm{a}}$ \\
\hline Intensity & $100.12 \pm 19.03$ & $119.03 \pm 17.23$ & $131.61 \pm 18.04$ & $0.002^{\mathrm{a}}$ \\
\hline Long-run emphasis & $1.19 \pm 0.08$ & $1.20 \pm 0.08$ & $1.19 \pm 0.05$ & 0.906 \\
\hline Total run length & $3433.29 \pm 808.54$ & $4140.06 \pm 1065.69$ & $5171.36 \pm 982.90$ & $0.002^{\mathrm{a}}$ \\
\hline T1 homogeneity & $0.006 \pm 0.0004$ & $0.0008 \pm 0.001$ & $0.007 \pm 0.0003$ & $<0.001^{\mathrm{a}}$ \\
\hline
\end{tabular}

Abbreviation: ANOVA, analysis of variance.

astatistically significant.

Table 2 Correlation of tumor size with nuclear morphometry.

\begin{tabular}{|l|l|l|l|}
\hline Morphometric parameter & $\begin{array}{l}\text { Tumor size }<\mathbf{5 m} \\
\boldsymbol{n}=\mathbf{3 8}\end{array}$ & $\begin{array}{l}\text { Tumor size } \mathbf{2} \mathbf{c m} \\
\boldsymbol{n}=\mathbf{7}\end{array}$ & $\begin{array}{l}\boldsymbol{p} \text {-Value } \\
\text { (independent sample } \boldsymbol{t} \text {-test) }\end{array}$ \\
\hline Nuclear area & $91.92 \pm 18.48$ & $112.99 \pm 12.99$ & $0.006^{\mathrm{a}}$ \\
\hline Perimeter & $35.38 \pm 4.95$ & $40.82 \pm 1.69$ & $0.006^{\mathrm{a}}$ \\
\hline Shape & $1.08 \pm 0.04$ & $1.11 \pm 0.03$ & 0.076 \\
\hline Long axis & $13.39 \pm 1.53$ & $14.75 \pm 0.59$ & $0.025^{\mathrm{a}}$ \\
\hline Short axis & $9.12 \pm 1.20$ & $10.31 \pm 0.46$ & $0.013^{\mathrm{a}}$ \\
\hline Intensity & $114.76 \pm 20.19$ & $135.85 \pm 7.04$ & $0.009^{\mathrm{a}}$ \\
\hline Long-run emphasis & $1.19 \pm 0.08$ & $1.20 \pm 0.08$ & 0.782 \\
\hline Total run length & $4095.59 \pm 1160.11$ & $4946.06 \pm 710.60$ & 0.068 \\
\hline T1 homogeneity & $0.0071 \pm 0.0012$ & $0.0078 \pm 0.0015$ & 0.252 \\
\hline
\end{tabular}

aStatistically significant.

Table 3 Correlation of lymph node status (positive/negative) with nuclear morphometry.

\begin{tabular}{|l|l|l|l|}
\hline Morphometric parameter & $\begin{array}{l}\text { Lymph node status negative } \\
\boldsymbol{n}=\mathbf{1 5}\end{array}$ & $\begin{array}{l}\text { Lymph node status positive } \\
\boldsymbol{n}=\mathbf{3 0}\end{array}$ & $\begin{array}{l}\boldsymbol{p} \text {-Value } \\
\text { (independent sample } \boldsymbol{t} \text {-test) }\end{array}$ \\
\hline Nuclear area & $76.65 \pm 12.46$ & $104.47 \pm 14.81$ & $<0.001^{\text {a }}$ \\
\hline Perimeter & $30.49 \pm 3.29$ & $39.10 \pm 2.67$ & $<0.001^{\text {a }}$ \\
\hline Shape & $1.05 \pm 0.02$ & $1.11 \pm 0.03$ & $<0.001^{\text {a }}$ \\
\hline Long axis & $11.92 \pm 0.98$ & $14.44 \pm 0.89$ & $<0.001^{\text {a }}$ \\
\hline Short axis & $8.06 \pm 0.84$ & $9.92 \pm 0.79$ & $<0.001^{\text {a }}$ \\
\hline Intensity & $98.19 \pm 12.18$ & $127.97 \pm 15.60$ & $<0.001^{\text {a }}$ \\
\hline Long-run emphasis & $1.16 \pm 0.05$ & $1.21 \pm 0.08$ & $0.019^{\text {a }}$ \\
\hline Total run length & $3260.32 \pm 818.74$ & $4711.66 \pm 958.68$ & $<0.001^{\text {a }}$ \\
\hline T1 homogeneity & $0.0064 \pm 0.0008$ & $0.008 \pm 0.001$ & $0.003^{\text {a }}$ \\
\hline
\end{tabular}

aStatistically significant.

The histopathological examination of 42 cases showed IDC (NOS) and 3 cases (6.7\%) of DCIS. Among the 42 cases of IDC (NOS), there were 6,27 , and 9 cases in grades 1,2 , and 3 , comprising $13.3,60$, and $20 \%$, respectively. On correlation of DCIS and histopathological grades 1 to 3 with morphometry, it was found that all the parameters except long-run emphasis, that is, nuclear area, perimeter, shape, long and short axes, intensity, total run length, and T1 homogeneity were highly significant on statistical analysis with all having $p<0.001$ (- Table 5).

\section{Discussion}

This study was planned to evaluate the role of nuclear morphometry vis-à-vis various clinicopathologic parameters in breast cancer. We intended to evaluate each of these criteria and also explore the move from subjectivity to quantified objectivity in breast cancer diagnosis and prognostication.

Studies have found interactive computerized nuclear morphometry to be an efficient and successful tool in distinguishing between cases of benign and malignant breast disease. 
Table 4 Correlation of number of mitosis with nuclear morphometric parameters.

\begin{tabular}{|l|l|l|l|}
\hline Morphometric parameter & $\begin{array}{l}\text { Mitosis }<6 \\
(\boldsymbol{n}=\mathbf{3 3})\end{array}$ & $\begin{array}{l}\text { Mitosis } \geq 6 \\
(\boldsymbol{n}=12)\end{array}$ & $\begin{array}{l}\boldsymbol{p} \text {-Value } \\
\text { (independent sample } \boldsymbol{t} \text {-test) }\end{array}$ \\
\hline Nuclear area & $87.86 \pm 14.30$ & $115.39 \pm 16.69$ & $<0.001^{\mathrm{a}}$ \\
\hline Perimeter & $34.72 \pm 4.58$ & $40.36 \pm 3.68$ & $<0.001^{\mathrm{a}}$ \\
\hline Shape & $1.08 \pm 0.03$ & $1.12 \pm 0.028$ & $0.002^{\mathrm{a}}$ \\
\hline Long axis & $13.11 \pm 1.36$ & $14.93 \pm 1.04$ & $<0.001^{\mathrm{a}}$ \\
\hline Short axis & $8.96 \pm 1.12$ & $10.24 \pm 0.86$ & $<0.001^{\mathrm{a}}$ \\
\hline Intensity & $111.89 \pm 18.49$ & $134.96 \pm 14.69$ & $0.003^{\mathrm{a}}$ \\
\hline Long-run emphasis & $1.19 \pm 0.08$ & $1.21 \pm 0.07$ & 0.482 \\
\hline Total run length & $3849.13 \pm 995.71$ & $5269.46 \pm 832.49$ & $<0.001^{\mathrm{a}}$ \\
\hline T1 homogeneity & $0.0073 \pm 0.001$ & $0.0071 \pm 0.0012$ & 0.510 \\
\hline
\end{tabular}

aStatistically significant.

Table 5 Correlation of histological grades with nuclear morphometry.

\begin{tabular}{|l|l|l|l|l|l|}
\hline $\begin{array}{l}\text { Morphometric } \\
\text { parameter }\end{array}$ & $\begin{array}{l}\text { DCIS } \\
(\boldsymbol{n}=\mathbf{3})\end{array}$ & $\begin{array}{l}\text { Histopathology } \\
\text { grade } \mathbf{1}(\boldsymbol{n}=\mathbf{6})\end{array}$ & $\begin{array}{l}\text { Histopathology } \\
\text { grade 2 }(\boldsymbol{n}=\mathbf{2 7})\end{array}$ & $\begin{array}{l}\text { Histopathology } \\
\text { grade 3 }(\boldsymbol{n}=\mathbf{9})\end{array}$ & $\begin{array}{l}\boldsymbol{p} \text {-Value } \\
\text { (one-way ANOVA test) }\end{array}$ \\
\hline Nuclear area & $85.63 \pm 5.10$ & $69.81 \pm 8.08$ & $93.08 \pm 12.78$ & $118.9 \pm 14.63$ & $<0.001^{\text {a }}$ \\
\hline Perimeter & $32.37 \pm 2.15$ & $29.58 \pm 4.36$ & $35.35 \pm 3.94$ & $40.19 \pm 4.31$ & $<0.001^{\text {a }}$ \\
\hline Shape & $1.04 \pm 0.005$ & $1.05 \pm 0.01$ & $1.09 \pm 0.03$ & $1.11 \pm 0.03$ & $<0.001^{\text {a }}$ \\
\hline Long axis & $12.02 \pm 0.59$ & $11.64 \pm 1.01$ & $13.66 \pm 1.18$ & $15.12 \pm 0.70$ & $<0.001^{\text {a }}$ \\
\hline Short axis & $8.63 \pm 0.54$ & $7.72 \pm 0.72$ & $9.31 \pm 1.03$ & $10.54 \pm 0.43$ & $<0.001^{\text {a }}$ \\
\hline Intensity & $92.64 \pm 6.21$ & $100.62 \pm 20.80$ & $117.65 \pm 17.25$ & $135.33 \pm 10.95$ & $<0.001^{\text {a }}$ \\
\hline Long-run emphasis & $1.17 \pm 0.05$ & $1.20 \pm 0.09$ & $1.20 \pm 0.09$ & $1.19 \pm 0.05$ & 0.934 \\
\hline Total run length & $4043.24 \pm 209.25$ & $2836.74 \pm 496.43$ & $4136.50 \pm 996.54$ & $5446.82 \pm 752.46$ & $<0.001^{\text {a }}$ \\
\hline T1 homogeneity & $0.006 \pm 0.0006$ & $0.006 \pm 0.000$ & $0.008 \pm 0.001$ & $0.007 \pm 0.001$ & $<0.001^{\text {a }}$ \\
\hline
\end{tabular}

Abbreviations: ANOVA, analysis of variance; DCIS, ductal carcinoma in situ.

aStatistically significant.

Diagnostic cutoff values for mean nuclear area (MNA) have been proposed to distinguish between benign and malignant breast lesions. Imprint cytology has been combined with morphometric analysis and has yielded superior results compared with those obtained by imprint cytology and frozen section in breast cancer diagnosis. With morphometric analysis, it has been reported that there was a significant difference between the MNAs, nuclear perimeter, and nuclear diameter between benign and malignant tissues. Feret circle, a measure of ellipticity, was not significant. These parameters have been advocated to be used intraoperatively in imprint smears to distinguish benign from malignant and suspicious lesions. ${ }^{3,4}$

In this study, geometrical (nuclear area, perimeter, nuclear shape, long axis, short axis, and intensity) and textural (long-run emphasis for coarseness of nuclear chromatin, total run length measuring proportion of coarse to fine chromatin, and T1 homogeneity indicating homogeneity of chromatin distribution) parameters were compared with clinicopathologic ones including cytological grade, tumor size, lymph node status, mitotic count, and histological grade and this association was subjected to statistical analysis.

The role of cytologic grading and its significance in prognostication has been highlighted in the published literature. Cytologic grading as a prognosticator of invasive breast carcinoma has been studied and compared with histomorphologic grading with reference to lymph node metastasis. Cytology was reported to be $89.1 \%$ sensitive and $100 \%$ specific as compared with histopathology for grading breast carcinoma. It was also found to be comparable to histologic grading and useful in providing information on the aggressiveness and tumor behavior of invasive ductal carcinoma of breast. ${ }^{5}$

The value of automated quantitative three-dimensional nuclear morphometry as an objective tool to enable development of sensitive and specific nuclear grade classification in breast cancer diagnosis has been documented. Abnormal cell nuclei have been found to have more nucleoli, markedly higher density, and clumpier chromatin organization compared with normal ones. ${ }^{6}$

In the present study, cytologic grades were correlated with nuclear morphometry. Barring long-run emphasis, all the other parameters, namely, nuclear area, perimeter, shape, long axis, short axis, intensity, total run length, and $\mathrm{T} 1$ homogeneity, were highly significant on statistical analysis with $p<0.05$.

Cytologic grading has been reported to have a high correlation with histologic grading and it is found to improve further on supplementation with image morphometric parameters, that is, nuclear diameter, nuclear area, nuclear roundness, nuclear perimeter, and gray level to compare with chromatin texture. ${ }^{7}$ This is in agreement with the present study.

Nuclear and histologic grade, lymph node status, tumor size, mitotic activity index, cellularity index, and mean and SD of nuclear area have been reported to be the most important single predictors of prognosis in breast carcinoma. 
Morphometry significantly adds to the prognosis prediction of lymph node status and tumor size. MNA has been reported to be significantly higher in tumors of the postmenopausal than premenopausal, in $\mathrm{LN}+$ than $\mathrm{LN}$ - patients, and in tumors over $3 \mathrm{~cm}$ than smaller ones. Significant differences between different clinical stages, histological grades, and histological types of tumors have been reported. Significant correlations have been reported between MNA and histological grade, standard mitotic index, and tumor size.,9

In the present study, the comparison of tumor size with morphometry yielded significant results only for geometric parameters, that is, nuclear area, perimeter, long and short axes, and intensity with $p<0.05$. All the geometric as well as textural parameters, that is, nuclear area, perimeter, shape, long and short axes, intensity, long-run emphasis, total run length, and $\mathrm{T} 1$ homogeneity were, on the other hand, found to be significantly associated with lymph node status (positive/negative). We found mitotic count to have a significant statistical association with all the geometric parameters including nuclear area, perimeter, shape, long axis, short axis, and intensity, with only one textural parameter, that is, total run length, signifying proportion of coarse to fine chromatin being significantly associated. This is majorly in concurrence with the published literature.

The histopathological examination showed IDC (NOS) in 42 cases and DCIS in 3 cases (6.7\%) in our study. Among the 42 cases of IDC (NOS), there were 6, 27, and 9 cases in histopathological grades 1,2 , and 3 , comprising $13.3,60$, and $20 \%$, respectively. On correlation of DCIS and histopathological grades 1 to 3 with morphometry, it was found that all the geometric and most of the textural parameters except long-run emphasis, that is, nuclear area, perimeter, shape, long and short axes, intensity, total run length, and $\mathrm{T} 1$ homogeneity were highly significant on statistical analysis with all having $p<0.001$.

Morphometric characteristics of different types and grades of breast cancer have been compared. Tumor area, circumference, maximal radius, minimal radius, convexity, length, width, elongation, nucleus/cytoplasm ratio, and shape factor (SHF) have been found to have significant differences among histological grades. Morphometric parameters have shown significant individual correlation with tumor type and grade, whereby the nuclear and cellular area, convexity, and circumference were found to be most significant. ${ }^{6}$

Nuclear morphometric features including MNA, SD of nuclear area (SDNA), mean nuclear perimeter (MNP), SD of nuclear perimeter (SDNP), and SHF have been used to identify aggressive tumor phenotype and provide additional prognostic information for patients with male breast carcinoma. These were compared with tumor histological grade, size, nodal status, deoxyribonucleic acid (DNA) ploidy evaluated by flow cytometry and cell proliferative activity. Comparison was also made with the immunohistochemical detection of p53, bcl-2, c-erbB-2, and c-myc proteins. Significant association was found between nuclear morphometric parameters and tumor grade, DNA content, and cell proliferation indices. SDNA was greater in p53-positive and bcl-2-negative cases; SDNP was greater in p53-positive cases; and SHF was lower in p53- and c-myc-positive cases. Overall survival was shorter in carcinomas with high MNA, SDNA, MNP, and SDNP and low SHF. ${ }^{10}$

In a study on correlation of nuclear perimeter, nuclear area, Feret ratio, and Feret circle in DCIS with tumor size, nuclear grade, necrosis, cell polarization, and architectural pattern, a statistically significant correlation was found between nuclear perimeter and area with all the pathologic parameters, with the strongest association observed for nuclear grade. Higher grade nuclei, DCIS with necrosis, comedo architecture rather than papillary, and absence of polarization were associated with larger nuclear area and perimeter. There was direct correlation for tumor size with nuclear area and perimeter. Nuclear roundness or lack of it did not factor as a significant component in the pathologic assessment. ${ }^{11}$

\section{Conclusion}

Based on this study, one can surmise that morphometry as a technique, holds immense promise in prognostication in breast carcinoma. It carries the added advantage of being objective and thus is free of individual biases. More studies need to be conducted to further evaluate the strength of this association and its utility in clinical practice.

\section{Conflict of Interest}

The authors declare no conflicts of interest.

\section{References}

1 Pienta KJ, Coffey DS. Correlation of nuclear morphometry with progression of breast cancer. Cancer 1991;68(9):2012-2016

2 Robinson IA, McKee G, Nicholson A, et al. Prognostic value of cytological grading of fine-needle aspirates from breast carcinomas. Lancet 1994;343(8903) :947-949

3 Abdalla F, Boder J, Buhmeida A, Hashmi H, Elzagheid A, Collan Y. Nuclear morphometry in FNABs of breast disease in Libyans. Anticancer Res 2008;28(6B) :3985-3989

4 Aggarwal G, Singh S, Marwah S, Duhan A, Mathur SK, , Marwah N, et al. Morphometric analysis in breast lesions a rapid conjunct to intraoperative imprint smears. Middle East J Cancer 2012;3(1):1-8

5 Khan N, Afroz N, Rana F, Khan M. Role of cytologic grading in prognostication of invasive breast carcinoma. J Cytol 2009;26(2):65-68

6 Prvulović I, Kardum-Skelin I, Sustercić D, Jakić-Razumović J, Manojlović S. Morphometry of tumor cells in different grades and types of breast cancer. Coll Antropol 2010;34(1):99-103

7 Tahlan A, Nijhawan R, Joshi K. Grading of ductal breast carcinoma by cytomorphology and image morphometry with histologic correlation. Anal Quant Cytol Histol 2000;22(3):193-198

8 Baak JPA, Van Dop H, Kurver PHJ, Hermans J. The value of morphometry to classic prognosticators in breast cancer. Cancer 1985;56(2):374-382

9 Ikpatt OF, Kuopio T, Collan Y. Nuclear morphometry in African breast cancer. Image Anal Stereol 2011;21(2):145

10 Chiusa L, Margaria E, Pich A. Nuclear morphometry in male breast carcinoma: association with cell proliferative activity, oncogene expression, DNA content and prognosis. Int J Cancer 2000;89(6):494-499

11 Tan PH, Goh BB, Chiang G, Bay BH. Correlation of nuclear morphometry with pathologic parameters in ductal carcinoma in situ of the breast. Mod Pathol 2001;14(10):937-941 